\title{
MEASUREMENT OF THE NONLINEAR ELASTICITY OF DOPED BULK-MODE MEMS RESONATORS
}

\author{
Yushi Yang ${ }^{l}$, Eldwin J. Ng ${ }^{l}, V u$ A. Hong ${ }^{l}$, Chae Hyuck Ahn ${ }^{l}$, Yunhan Chen ${ }^{l}$,Elia Ahadi, \\ Mark Dykman', and Thomas W. Kenny ${ }^{l}$ \\ ${ }^{1}$ Stanford University, California, USA \\ ${ }^{2}$ Michigan State University, East Lansing, Michigan, USA
}

\begin{abstract}
In this paper, we present measurements of the nonlinear amplitudefrequency (A-f) coefficients for three types of bulk-mode silicon MEMS resonators with different doping type and concentration. Our experimental results clearly show a strong dependence of the nonlinearity character on the orientation, doping level, and vibrational mode shape. These results indicate that the doping can have a significant impact on the character and strength of the elastic nonlinearities in MEMS devices.
\end{abstract}

\section{INTRODUCTION}

High performance MEMS oscillators have gained much attention as an alternative for quartz since they offer potential benefits such as volume and cost reduction, low power consumption, and IC compatibility [1, 2]. The use of MEMS oscillators in wireless communication requires device performance characteristics such as high stability, high quality factor (Q), and large power handing capability. These will provide a larger signalto-noise ratio and reduced phase noise [3]. However, because the power handling capability of MEMS oscillators is inversely related to the resonator size, oftentimes the device has to be driven into nonlinear regime to obtain suitable signal-to-noise ratio and performance [4].

Another aspect that has gained wide attention is frequencytemperature compensation. Recent research has demonstrated the use of heavily doped $\mathrm{p}$ - or n-type silicon for passive frequencytemperature compensation $[5,6]$, thus making it attractive for this study. Adding dopants to the silicon lattice will not only introduce free charge carriers, but will also cause a strain in the lattice due to a size dissimilarity between the silicon and the dopant atoms. These effects shift the semiconductor energy bands and result in a change in the material elastic constants, since any change in the total electronic energy content of the crystal will also contributes to the total elastic energy [7]. Nonlinearities in lightly doped silicon resonators have been studied [8], and the effect has also been noted in heavily doped silicon [9]. This examines the nonlinear effects for a variety of doping types/levels and devices, showing that substantial changes in the strength and character of the nonlinearity should be expected in doped silicon resonators

One common way of characterizing the nonlinear behavior of a resonator is to analyze the amplitude-frequency effect (A-f effect). For nonlinear analysis, correction terms are added to the equation of motion (EOM) of the resonator, which yields:

$$
m \ddot{x}+2 \lambda \dot{x}+k_{0} x+k_{1} x^{2}+k_{2} x^{3}=F \cos (2 \pi f t)
$$

where $k_{1}$ and $k_{2}$ are the $2^{\text {nd }}$ and $3^{\text {rd }}$ order nonlinear forcing terms respectively. The linear natural frequency is defined as $f_{0}=$ $\frac{1}{2 \pi} \sqrt{k_{0} / m}$. Analytically it has been shown that the peak frequency is a function of the peak amplitude in the presence of the nonlinear terms [10]:

$$
f_{0}{ }^{\prime}=f_{0}+\kappa X_{0}^{2}
$$

where $X_{0}$ is the resonator peak amplitude, $f_{0}{ }^{\prime}$ is the shifted frequency at large displacement, and $\kappa$ is the A-f coefficient, which is calculated as:

$$
\kappa=\left(\frac{3 k_{2}}{8 k_{0}}-\frac{5 k_{1}^{2}}{12 k_{0}^{2}}\right) f_{0}
$$

In this paper, we present the modeling and experimental observation of how doping affects the devices' nonlinear behavior.

\section{DESIGN AND FABRICATION}

Three types of bulk-mode resonators are designed and fabricated (See Table.1 for resonator dimensions): length extensional mode resonator $(\boldsymbol{L E})$, Lamé mode resonator (Lamé), and dual breathe mode ring resonator (Ring). Nonlinearities in capacitive resonators are usually classified into electrostatic and mechanical (geometric and material). Compared with flexural-mode resonators, bulk-mode resonators are usually dominated by material nonlinearity effects, thus making them suitable candidates for studying the effect of doping [8].
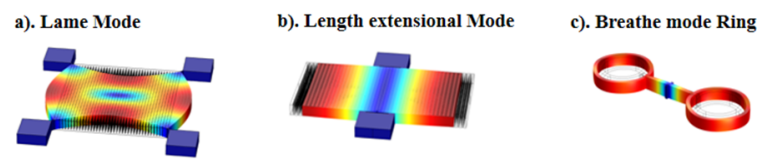

Figure 1: Mode shape of the three types of bulk-mode resonators

Four different types of doping levels are investigated, with the measured device layer resistivity listed as follows: Phosphorus (NP) $1.78 \mathrm{~m} \Omega$-cm, Arsenic (NA) $3.1 \mathrm{~m} \Omega$-cm, Antimony (NS) $17.1 \mathrm{~m} \Omega-$ $\mathrm{cm}$, and Boron $(\boldsymbol{P B}) 15.8 \mathrm{~m} \Omega-\mathrm{cm}$. These doping densities are representative of the characteristics of commercially-available silicon-on-insulator (SOI) wafers

The devices are fabricated using a wafer-level encapsulation process (epi-seal). The process starts off with (100) SOI wafer with a $40 \mu \mathrm{m}$ single-crystal device layer doped as described above, and a $2 \mu \mathrm{m}$ buried oxide layer. First, deep reactive ion etching is used to etch the device structural outline. A $2 \mu \mathrm{m}$ sacrificial oxide layer is then deposited and electrical contacts to the device layer are etched. Afterwards, the first epitaxial polysilicon cap layer is deposited. Vent holes are then etched and the resonant structures are released using vapor phase HF. Immediately after the release, the second polysilicon cap layer, which acts as the final encapsulation layer is deposited. Residual hydrogen is then diffused out to create a low pressure cavity $(<1 \mathrm{~Pa})$. Finally, electrical contacts are etched and aluminum is deposited and patterned. This process provides a clean and low pressure environment, which allows the devices to achieve long-term stability [11].

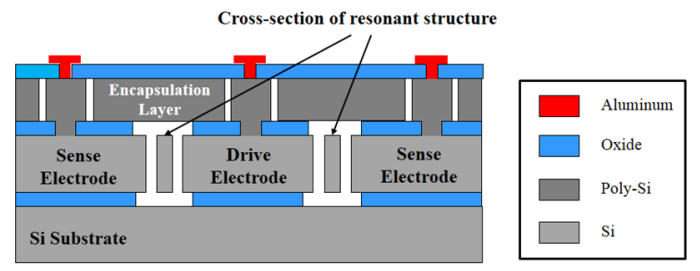

Figure 2: Final cross section view of the epi-seal process 


\section{RESONATOR MODELING}

In this section, we present lumped mass models following the method given in [8] to analyze the nonlinear dynamics of these continuum systems. To begin, the resonators are transformed into single degree-of-freedom systems with the lumped EOM given as:

$$
m_{\text {eff }} \ddot{X}+c \dot{X}+k(X) X=F_{\text {tot }}
$$

where $X$ is the maximum resonator displacement, $m_{e f f}$ is the effective mass, $c$ is the linear damping coefficient, $k(X)$ represents the spring constants, and $F_{\text {tot }}$ is the total electrostatic driving force. The effective mass of the resonators are simulated using COMSOL as:

$$
m_{\text {eff }}=\iiint_{V} \rho\left(\frac{u}{x}\right)^{2} d V
$$

Here the resonator's displacement field $u$ is first normalized with respect to the resonator's maximum displacement, followed by an integration across the entire resonator volume. Accounting for a lateral blowout of $0.1 \mu \mathrm{m}$ during the definition etch, the simulated results are listed in Table.1.

\section{Table 1: Resonator dimensions and other design parameters}

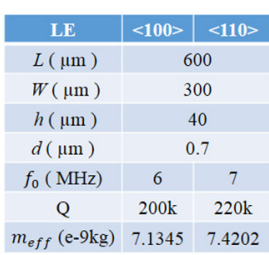

* $L$ : Resonator length

\begin{tabular}{|c|c|c|}
\hline Lame & $\langle 100\rangle$ & $\langle 110\rangle$ \\
\hline$L(\mu \mathrm{m})$ & \multicolumn{2}{|c|}{400} \\
\hline$h(\mu \mathrm{m})$ & \multicolumn{2}{|c|}{40} \\
\hline$d(\mu \mathrm{m})$ & \multicolumn{2}{|c|}{0.7} \\
\hline$f_{0}(\mathrm{MHz})$ & 8 & 10 \\
\hline Q & $120 \mathrm{k}$ & $80 \mathrm{k}$ \\
\hline$m_{e f f}(\mathrm{e}-9 \mathrm{~kg})$ & 7.0630 & 7.1677 \\
\hline
\end{tabular}

$* d$ : Designed gap size

\begin{tabular}{|c|c|}
\hline Ring & $<110>$ \\
\hline$R_{\text {in }}(\mu \mathrm{m})$ & 59 \\
\hline$R_{\text {out }}(\mu \mathrm{m})$ & 69 \\
\hline$h(\mu \mathrm{m})$ & 40 \\
\hline$d(\mu \mathrm{m})$ & 1 \\
\hline$f_{0}(\mathrm{MHz})$ & 19.7 \\
\hline $\mathrm{Q}$ & $240 \mathrm{k}$ \\
\hline$m_{\text {eff }}(\mathrm{e}-9 \mathrm{~kg})$ & 0.4596 \\
\hline
\end{tabular}

* $h$ : Resonator height $* R_{\text {out }}:$ Ring outer radius
To account for the nonlinear effects, higher order correction terms are incorporated into the spring constant model:

$$
k(X)=\left(k_{0 m}+k_{0 e}\right)+\left(k_{1 m}+k_{1 e}\right) X+\left(k_{2 m}+k_{2 e}\right) X^{2}
$$

For electrostatically driven resonators, the spring constants include both mechanical and electrical terms due to the electrostatic softening effect [10].

The electrical contribution to the spring constants can be found via studying the electrostatic driving force:

$$
F=\frac{\partial U}{\partial X}=\frac{\Delta V^{2}}{2} \frac{\partial C_{t o t}}{\partial X}
$$

where $\Delta V$ is the potential difference across the electrodes and $C_{t o t}$ is the total capacitance. Based on the electrode placement and the corresponding driving/sensing method (i.e, fully differential), the electrical spring constants for the three types of resonators can be estimated, assuming that the ac drive voltage $\left(V_{a c}\right)$ is significantly less than the bias voltage $\left(V_{\text {bias }}\right)$, and electrodes remain as parallel plates.

To account for the mechanical nonlinearities, a nonlinear strain-dependent modulus is needed to solve for the EOM. For the LE and Ring resonators, a nonlinear Young's modulus is taken into account:

$$
E=E_{0}+E_{1} \gamma+E_{2} \gamma^{2}
$$

where $\gamma$ is the engineering strain. Substituting the nonlinear Young's modulus into the wave equation and integrating over the mode shape, the nonlinear spring constants can be obtained.

- LE: $k_{0 m}=\frac{\pi^{2} E_{0} A_{0}}{2 L}, k_{1 m}=\frac{4 \pi^{2} E_{1} A_{0}}{3 L^{2}}, k_{2 m}=\frac{3 \pi^{4} E_{2} A_{0}}{8 L^{3}}$

The Lamé mode resonator on the other hand, can be considered as a pure shear structure, therefore to simplify the calculation, a nonlinear shear modulus should be introduced.

$$
G=G_{0}+G_{1} \gamma+G_{2} \gamma^{2}
$$

The calculated mechanical spring constants incorporating the nonlinear material effects can be found:

- Lame: $k_{0 m}=\pi^{2} G_{0} h, k_{1 m}=0, k_{2 m}=\frac{9 \pi^{4} G_{2} h}{4 L^{2}}$

\section{EXPERIMENT CHARACTERIZATION}

For all experiments, the resonators were tested in an oven at a temperature of $40 \pm 0.2^{\circ} \mathrm{C}$ to eliminate temperature drift effects. To operate the devices, a dc polarization voltage was applied to the resonant structure and an ac drive voltage was applied to the drive electrodes. The output current generated by the motion of the resonator is transformed into a voltage signal and amplified by an off-chip trans-impedance amplifier.

Two types of experiments were performed to characterize the nonlinear behavior of these devices: open-loop frequency sweeps with bias voltage variations and closed-loop experiments for operation beyond the critical bifurcation limit.

\section{Open-loop Electrostatic Frequency Tuning}

For electrostatic resonators, knowing the gap size is crucial before any analysis can take place. However, due to fabrication uncertainties, the actual gap size may vary from the layout design. This will introduce errors if one uses the design gap size (Table 1) in the data analysis. In order to have a better estimation of the effective gap size, the electrostatic softening effect can be utilized. Due to the electrostatic softening effect, the resonant frequency will decrease as $V_{\text {bias }}$ increases (Fig. 3).
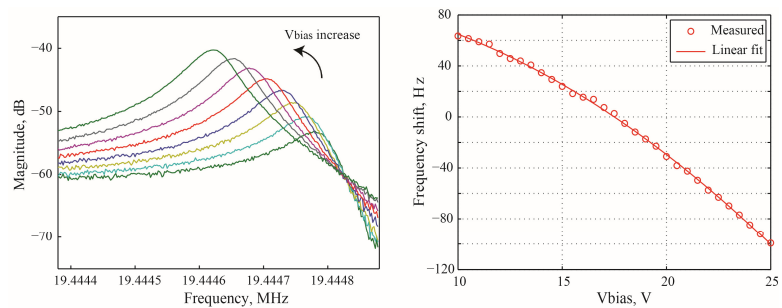

Figure 3: Example of the frequency tuning effect for ring resonator.

\begin{tabular}{|c|c|c|c|c|c|c|c|c|c|c|}
\hline \multirow{2}{*}{$\begin{array}{l}\text { Dopant } \\
\text { type }\end{array}$} & \multicolumn{2}{|c|}{$L E<100>$} & \multicolumn{2}{|c|}{$\mathrm{LE}<110>$} & \multicolumn{2}{|c|}{ Lame $\langle 100\rangle$} & \multicolumn{2}{|c|}{ Lame $\langle 110\rangle$} & \multicolumn{2}{|c|}{ Ring } \\
\hline & $k_{0 m}(\mathbf{N} / \mathbf{m})$ & $d(\mu \mathrm{m})$ & $k_{0 m}(\mathbf{N} / \mathbf{m})$ & $d(\mu \mathrm{m})$ & $k_{0 m}(\mathbf{N} / \mathbf{m})$ & $d(\mu \mathrm{m})$ & $k_{0 m}(\mathrm{~N} / \mathrm{m})$ & $d(\mu \mathrm{m})$ & $k_{0 m}(\mathrm{~N} / \mathrm{m})$ & $d(\mu \mathrm{m})$ \\
\hline NP & $9.9174 \mathrm{e}+06$ & 0.941 & $1.3997 \mathrm{e}+07$ & 0.904 & $1.7338 \mathrm{e}+07$ & 1.059 & $2.7920 \mathrm{e}+07$ & 0.805 & $6.9559 \mathrm{e}+06$ & 1.407 \\
\hline NA & $1.0074 \mathrm{e}+07$ & 0.991 & $1.4083 \mathrm{e}+07$ & 0.912 & $1.7661 \mathrm{e}+07$ & 1.088 & $2.7955 \mathrm{e}+07$ & 0.955 & $6.8603 e+06$ & 1.332 \\
\hline NS & $1.0338 \mathrm{e}+07$ & 0.882 & $1.4233 \mathrm{e}+07$ & 0.841 & $1.8223 \mathrm{e}+07$ & 0.860 & $2.8341 \mathrm{e}+07$ & 0.828 & $7.0838 \mathrm{e}+06$ & 1.360 \\
\hline PB & $1.0320 \mathrm{e}+07$ & 0.841 & $1.4189 \mathrm{e}+07$ & 0.809 & $1.8232 \mathrm{e}+07$ & 0.848 & $2.8300 \mathrm{e}+07$ & 0.796 & $7.0779 \mathrm{e}+06$ & 1.260 \\
\hline
\end{tabular}
The frequency decreases as V bias increases.

Neglecting the higher order electrostatic and mechanical softening/stiffening effects, the first order relation between $V_{\text {bias }}$ and resonant frequency $f_{0}$ can be calculated as:

Table 2: Fitted linear mechanical spring constant and gap size 


$$
f_{0}=\frac{1}{2 \pi} \sqrt{\frac{k_{0 m}-k_{0 e}}{m_{e f f}}}=\frac{1}{2 \pi} \sqrt{\frac{k_{0 m}-V_{b i a s}^{2} \epsilon_{0} A_{t o t} / d^{3}}{m_{e f f}}}
$$

where $A_{\text {tot }}$ is the total electrode area for the resonators. By substituting the simulated effective mass given in Table 1, and fitting the experimental data with Eqn.12, the fitted linear mechanical spring constant and gap size can be found (Table.2). It should be noted that the gap size obtained from the fitting is the effective gap size due to parallel plate assumption.

\section{Closed-loop Measurement}

The driving condition and stability criteria differs between the closed-loop and the open-loop measurement. In the open-loop experiment, the resonator is driven at a particular frequency, therefore instability can occur when the resonator is driven beyond the nonlinear bifurcation point. In the closed-loop setup, the feedback circuit sets a phase between the input and output of a resonator, allowing stable operation under any frequency and amplitude condition [4]. To create a variable-phase closed-loop feedback system, a Zurich HF2LI lock-in amplifier is used to track the resonant peak and fix the operating phase (Fig. 4). For each type of resonator, the DC bias voltage was held constant and the AC drive voltage was gradually increased until the resonant peak begins to show nonlinear behavior.

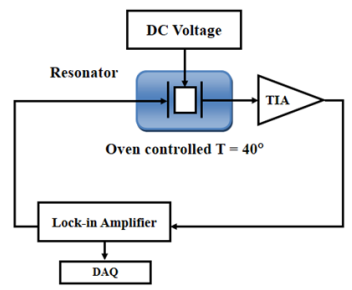

Figure 4: Close-loop experimental setup
The closed-loop frequency response plots for the different resonators are shown in Fig. 5. From Fig. 5, a few points can be observed:

1. For any particular type of resonator oriented along the same crystalline direction, the nonlinear behavior varies dramatically as doping changes. For example, the ring resonator shows a hardening effect for NP and NA doping, whereas for NS and PB doping, the resonator shows a softening effect.

2. For any particular type of resonator under the same doping condition, the nonlinearity is affected by device orientation. For example, the Lamé mode resonators oriented in the $<100>$ direction have a completely opposite nonlinear behavior than those oriented in the $<110>$ direction.

3. The nonlinearity also has strong correlations to the resonator's vibrational mode shape.

\section{DISCUSSION}

Following (2), the A-f coefficients have been extracted based on the above experimental results to further study the A-f effect.

$$
\kappa=\frac{f_{0}^{\prime}-f_{0}}{X_{0}^{2}}
$$

The resonator displacement can be back calculated from the measured voltage output following:

$$
X \cong \frac{V_{\text {out }} d^{2}}{2 \pi f_{0}^{\prime} G V_{\text {bias }} \epsilon_{0} W h}
$$

where $G$ is the gain of the trans-impedance amplifier. Fig.6 plots the extracted frequency shift versus displacement square for the resonators, with the fitted $\kappa$ listed in Table. 3 .

From the extracted results, the general trend is that the higher the n-type doping, the larger the absolute value of $\kappa$ for all the resonators tested, and a trend of $\left|\kappa_{N P}\right|>\left|\kappa_{N A}\right|>\left|\kappa_{N S}\right|>\left|\kappa_{P B}\right|$ can also be observed.

It should be note that the values reported in Table. 3 represents approximations to the A-f coefficient for four reasons:
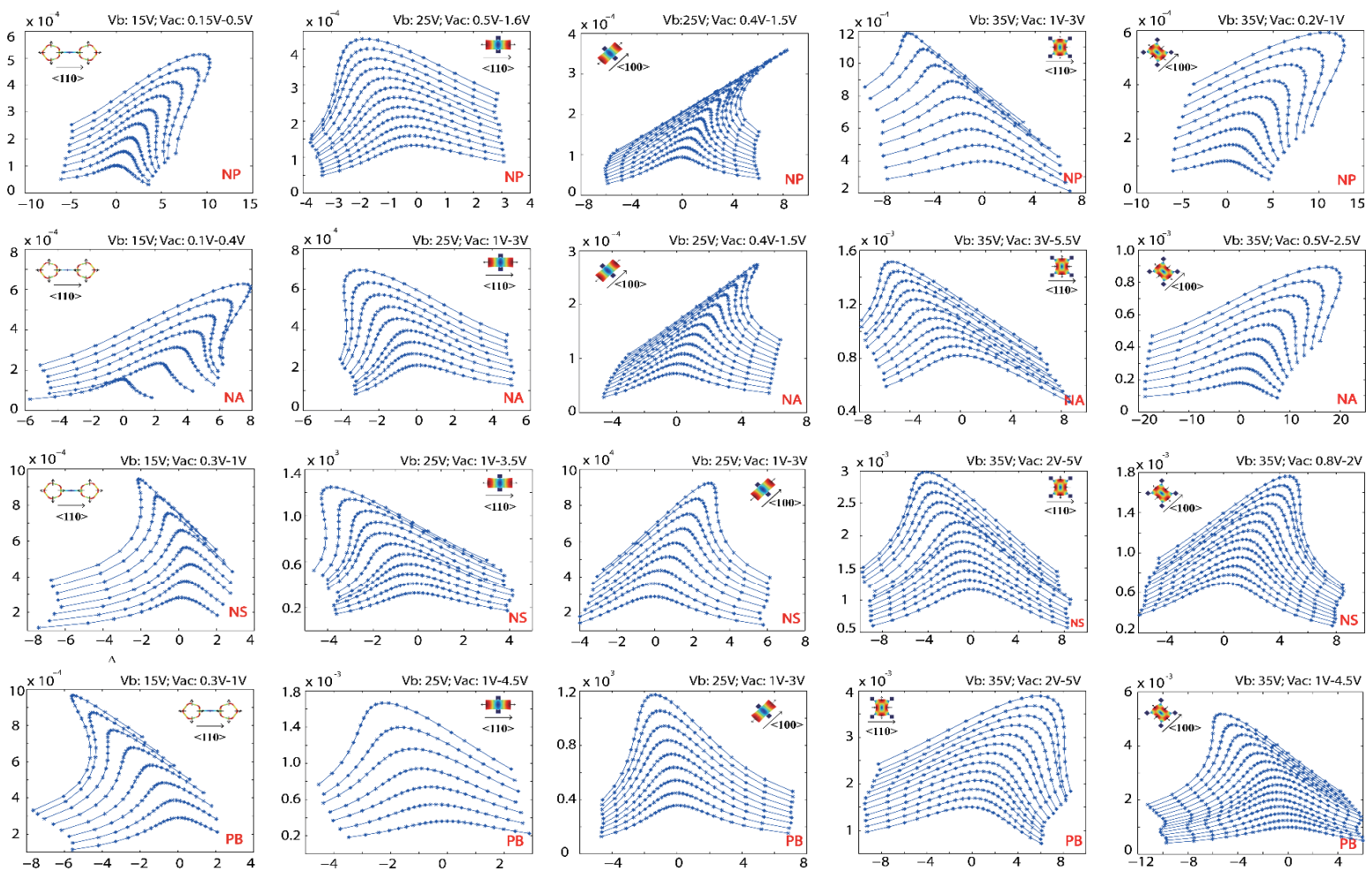

Figure 5: Measured close-loop amplitude frequency response curve for the three type of devices oriented in the $<100>$ and $<110>$ crystalline directions under different doping. For each plot, the $x$ axis is labeled $\Delta f / f_{0}$ in ppm, and the $y$ axis is the measured output voltage in Vrms. 

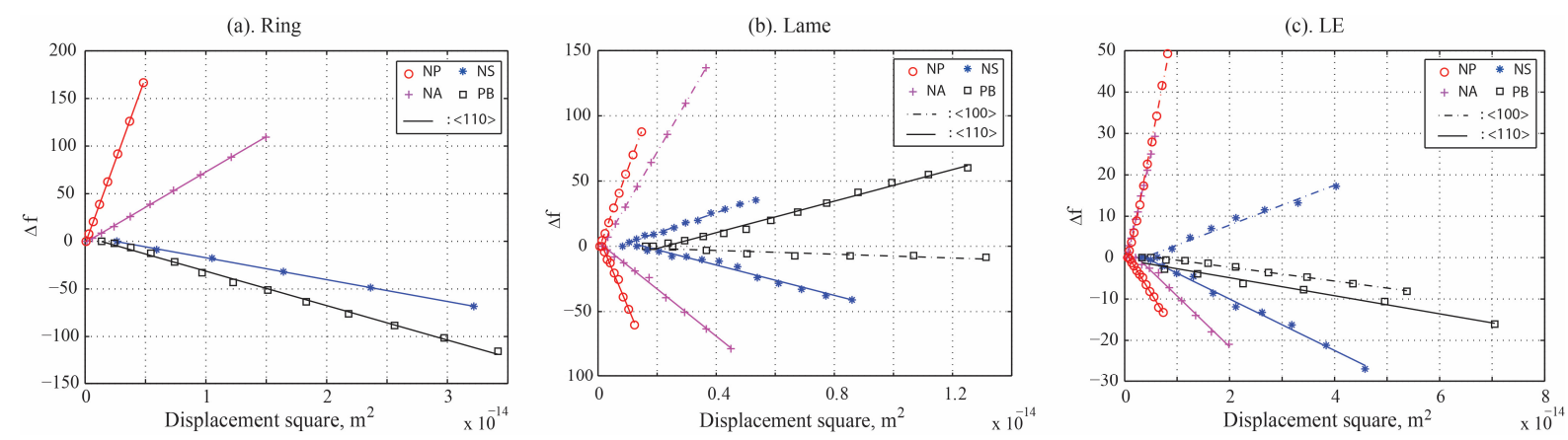

Figure 4: Plotted frequency shift vs. displacement square for different kind of resonators under various doping and orientation. Here the A-f coefficient $\kappa$ is represented by the slope of each line.

1. The parallel plate assumption neglects the displacement distribution effect due to the resonator mode shape and anchor location, which will contribute errors in terms of data extraction (i.e. Lamé mode resonator).

2. In order to release large areas in the epi-seal process, release holes must be added to the resonator body. This in turn may introduce certain errors when estimating certain parameters for the resonators (e.g. spring constants).

3. The resonator mode shape may not be correctly estimated. For example, the mode shape of the Ring resonator has strong dependence by the anisotropy of the silicon material, as well as the coupling beam length that connects the two rings. A mismatch between the coupling beam length and the ideal beam length may lead to distortion in the mode shape.

4. Electrostatic softening effects may come into play at higher bias voltages. If taking the $\mathrm{PB}$-doped Lame $<100>$ resonator as example, the fitted $\kappa$ is $-1.1475 \mathrm{e} 15 \mathrm{~Hz} / \mathrm{m}^{2}$, from this value the $3^{\text {rd }}$ order mechanical spring constant can be back calculated as $k_{3 m} \cong-7.1 e 15 \mathrm{~N} / \mathrm{m}^{3}$, while the $3^{\text {rd }}$ order electrical spring constant is found to be $k_{3 e} \cong-1.5 e 15 \mathrm{~N} / \mathrm{m}^{3}$.

Table 3: Fitted A-f coefficients

\begin{tabular}{|c|c|c|c|c|c|}
\hline \multirow{2}{*}{$\begin{array}{c}\mathbf{k} \\
\left(\mathrm{Hz} / \mathbf{m}^{2}\right)\end{array}$} & \multicolumn{2}{|c|}{$V_{\text {bias }}=25 \mathrm{~V}$} & \multicolumn{2}{|c|}{$V_{\text {bias }}=35 \mathrm{~V}$} & \multirow{2}{*}{$\begin{array}{c}V_{\text {bias }}=20 \mathrm{~V} \\
\text { Ring }<110>\end{array}$} \\
\hline & $\mathrm{LE}<100>$ & $L E<110>$ & Lame $<100>$ & Lame $<110>$ & \\
\hline NP & $6.4621 \mathrm{e}+15$ & $-2.0376 \mathrm{e}+15$ & $6.2551 \mathrm{e}+16$ & $-5.4349 \mathrm{e}+16$ & $3.4988 \mathrm{e}+16$ \\
\hline NA & $5.3959 e+15$ & $-1.2247 e+15$ & $3.9018 \mathrm{e}+16$ & $-1.9866 e+16$ & $7.4221 \mathrm{e}+15$ \\
\hline NS & $4.8171 \mathrm{e}+14$ & $-6.2194 \mathrm{e}+14$ & $7.7634 \mathrm{e}+15$ & $-5.8247 \mathrm{e}+15$ & $-2.2885 \mathrm{e}+15$ \\
\hline PB & $-1.6825 e+14$ & $-2.1780 e+14$ & $-1.1475 \mathrm{e}+15$ & $6.0437 e+15$ & $-3.6134 \mathrm{e}+15$ \\
\hline
\end{tabular}

\section{CONCLUSION}

In this paper, we have investigated the effect of doping on the nonlinear behavior of three types of bulk-mode silicon MEMS resonators. By utilizing the electrostatic frequency tuning effect, the effective electrode gap size can be obtained. Based on the closedloop amplitude frequency response measurement, the A-f coefficient can then be extracted. It can be seen that for a certain family of resonators, the device with the highest doping gives the largest absolute value of $\kappa$. A trend of the resonator's nonlinear performance can also be seen as the doping level varies. Our measurements clearly demonstrate that the resonators' nonlinear characteristics are strongly dependent on the crystal orientation, doping level, and operating mode shape. Future work will focus on utilizing these parameters to study the nonlinear elasticity for doped silicon.

\section{REFERENCES}

[1] C. Nguyen, "MEMS Technology for Timing and Frequency Control", IEEE Trans. Ultrasonics, Ferroelectrics and
Frequency Control, vol. 54, no. 2, pp. 251-270, Feb. 2007.

[2] J.T.M van Beek, et al, "A review of MEMS oscillators for frequency reference and timing applications", J. Micromech. Microeng, vol.22, no.1, 013001.

[3] M. Pardo, et al. "Phase Noise Shaping via Forced Nonlinearity in Piezoelectrically Actuated Silicon Micromechanical Resonators", MEMS 2011, Cancun, MEXICO.

[4] H.K. Lee, et al, "Stable operation of MEMS oscillators far above the critical vibration amplitude in the nonlinear regime", JMEMS, vol.20, no.6, 2010.

[5] A. K. Samarao, et al. "Temperature Compensation of Silicon Micromechanical Resonators via Degenerate Doping", IEDM 2009, pp. 789-792.

[6] E.J. Ng, et al. "Localized, degenerately doped epitaxial silicon for temperature compensation of resonant MEMS systems", Transducers 2013, pp. 2419-2422.

[7] J. J. Hall, "Electronic Effects in the Elastic Constants of n-Type Silicon", Physical Review, vol. 161, no. 3, 1967, pp. 756-761.

[8] V. Kaajakari, et al, "Nonlinear Limits for Single-crystal Silicon Microresonators", JMEMS, vol.13, no.5, 2004.

[9] M. Shahmohammadi, et al, "Nonlinearity reduction in silicon resonators by doping and re-orientation", MEMS 2013, Taipei, Taiwan.

[10] L. D. Landau, et al. "Resonance in non-linear oscillations", Mechanics, vol. 1, Course of Theoretical Physics, 3 ed., MA USA: Butterworth-Heinemann, pp. 87-92, 1982.

[11] B. Kim, et al, "Frequency stability of wafer-scale film encapsulated silicon based MEMS resonator", Sensors and Actuators A (Physical), vol. 136, no.1, pp.125-131, 2007.

[12] M. Agarwal, et al, "A study of electrostatic force nonlinearities in resonant structures", $A P L, 92,104106,2008$.

\section{ACKNOWLEDGEMENTS}

This work was supported by DARPA grant FA8650-13-17301, "Mesodynamic Architectures (MESO)," managed by Dr. Jeff Rogers. The fabrication work was performed at the Stanford Nanofabrication Facility (SNF) which is supported by National Science Foundation through the NNIN under Grant ECS-9731293. The authors would like to thank the staffs at SNF for their help during the fabrication process.

\section{CONTACT}

*Yushi Yang, tel: +1-765-404-0884; ysyang88@stanford.edu 